\title{
Confirmation of Hybridity using DNA-based Markers is Essential in Chickpea (Cicer arietinum L.)
}

\author{
Sanchit Thakur', Jai Dev Sharma ${ }^{2 *}$ and Kamal Dev Sharma ${ }^{3}$ \\ ${ }^{1}$ Department of Plant Breeding and Genetics, ${ }^{2}$ Department of Tea Husbandry \& Technology, \\ ${ }^{3}$ Department of Agricultural Biotechnology, CSKHPKV, Palampur, India \\ *Corresponding author
}

\section{A B S T R A C T}

\begin{tabular}{l} 
Ke y w o r d s \\
Chickpea, \\
$\begin{array}{l}\text { Hybridization, SSR, } \\
\text { True hybrids }\end{array}$ \\
\hline Article Info \\
\hline $\begin{array}{l}\text { Accepted: } \\
\text { 07 October } 2020 \\
\text { Available Online: } \\
10 \text { November } 2020\end{array}$ \\
\hline
\end{tabular}

Confirmation of hybridity is essential to exclude selfed plants in plant breeding programmes. The morphological markers for confirmation of hybridity in chickpea are unavailable in closely related germplasm and these results in ambiguity in identification of true hybrids. In present study, cold tolerant parent ICC-16349 (donor) and cold susceptible parent GPF-2 (recipient) were crossed to generate 80 putative hybrids. The parents were screened using 51 simple sequence repeat (SSR) markers, of which only one i.e TA 180 exhibited polymorphism. Screening of 80 putative hybrids using TA 180 revealed that only $34(42.5 \%)$ of the putative hybrids were true hybrids. The study indicated that in chickpea breeding programmes, hybridity must be confirmed using DNA based markers to avoid inclusion of selfed plants as hybrids.

\section{Introduction}

Chickpea (Cicer arietinum L.) $(2 \mathrm{n}=2 \mathrm{x}=16)$ is one of the important pulse crop that was first grown in Turkey about 7000 BC (Philologos, 2005). It is a self pollinated crop and belongs to kingdom Plantae, order Fabales, family Fabacae, genus Cicer and species arietinum. The crop is grown traditionally in semi-arid zones of Middle-East, Pakistan and India. According to Vavilov (1926), there are two primary centres of diversity of chickpea, namely, South-West Asia and Mediterranean while Ethiopia is designated as the secondary centre. The centre of origin of the crop is considered to be in South-Eastern Turkey and
Northern Syria where it is believed to be evolved from its progenitor Cicer reticulatum (Maesen, 1987). Chickpea is also known as Bengal gram, Garbanzo bean, Chana and Shonagalu in different chickpea producing areas of the world.

India which contributes to about $60 \%$ of the total world's production, is the largest producer and consumer of Chickpea (Varshney et al., 2014). In India, chickpea is mainly produced in Madhya Pradesh, Maharashtra, Rajasthan, Uttar Pradesh, Andhra Pradesh, Karnataka, Chhattisgarh, Bihar and Jharkhand and these states contribute more than $95 \%$ to the 
total production. India still imports it from other nations besides being the largest producer of chickpea due to low productivity which is due to the abiotic and biotic stresses and use of low yielding varieties.

Lack of diagnostic morphological markers for the confirmation of hybidity owing to insufficient genetic variability in cultivated chickpea species along with shortage of polymorphic markers is a major constraint for identification of true hybrids in chickpea (Atalay \& Babazogles, 2012) are the major hindrances to confirm hybridity in this crop. Consequently, there are chances of categorizing false hybrids as true hybrids leading to errors in the breeding programmes resulting in wastage of resources and time. At present, no information is available for the extent of inclusion of false hybrids in chickpea breeding programmes. For hybridity confirmation in chickpea, DNA-based markers may be the markers of choice to ascertain the hybrid nature. Among vast categories of DNA markers available for chickpea, simple sequence repeats (SSRs) are preferred due to codominant nature, locus specificity, high reproducibility and ease to use (Tautz and Renz, 1984). SSRs, also known as sequence tagged microsatellite site or microsatellite markers (Beckmann \& Soller, 1990) are widely used in genetic diversity analysis, population genetics, marker assisted selection and genetic mapping. Most of the important legumes in India including chickpea are accompanied by lack of genomic resources as limited SSR markers have been reported so far. SSR markers being codominant, detect alleles of both male and female parents and hence, are ideal for differentiation of true hybrids from the selfed individuals. The present study was formulated to estimate the extent of false positive hybrids in chickpea and to confirm the hybridity of $F_{1}$ plants by using SSR markers.

\section{Materials and Methods}

Hybridization was conducted between two parents having contrasting traits viz., cold tolerance (ICC-16349) and cold susceptibility (GPF-2) where 'ICC-16349' was selected as a donor and 'GPF-2' as a recipient. The seeds of hybrids and parents was sown in 10" diameter pots using a standard potting mixture (Soil: Sand: FYM: : 1: 1: 1) . At 3-4 leaf stage, a small amount of leaf tissue from each putative hybrid was harvested. The leaves were transported immediately in ice to lab for DNA extraction. The leaves were crushed in liquid nitrogen and DNA was isolated by using CTAB method (Murray and Thompson, 1980).

The DNA of parents was amplified by using a set of 51 SSR markers developed by Winter et al., (1999) and Gaur et al., (2011) (Table 1). Off the 51 SSR primers, only one i.e. TA 180 generated polymorphism between the parents. For polymerase chain reaction (PCR) assay, $10 \mu \mathrm{l}$ PCR reaction mixture was prepared which constituted $6.7 \mu \mathrm{l}$ sterile double distilled water, $1 \mu \mathrm{l} 10 \mathrm{X}$ Taq buffer, $0.3 \mu \mathrm{l}$ DNTPs $(2 \mathrm{mM}), 1.2 \mu \mathrm{l}$ DNA $(25-50 \mathrm{ng} / \mu \mathrm{l})$, $0.2 \mu \mathrm{l}$ DNA polymerase $(1 \mathrm{U} / \mu \mathrm{l})$ and $0.3 \mu \mathrm{l}$ each forward and reverse primer. PCR profile with initial denaturation of $5 \mathrm{~min}$ at $95^{\circ} \mathrm{C} ; 35$ cycles with denaturation at $94^{\circ} \mathrm{C}$ for 30 seconds, annealing temperature as per melting temperature of primer used, followed by extension at $72^{\circ} \mathrm{C}$ for $1 \mathrm{~min}$; and a final extension at $72^{\circ} \mathrm{C}$ for 8 mins. The amplification products were stored at $4^{0} \mathrm{C}$ and were resolved by gel electrophoresis in horizontal agrose system at $100 \mathrm{~V}$ for $2 \mathrm{hrs}$ staining using ethidium bromide $(0.5 \mu \mathrm{g} / \mathrm{ml})$ in $3 \%$ agarose gel ( $0.5 \mathrm{X}$ TAE Buffer). Gel documentation system was used to visualize the amplified products and size of the amplicon was estimated by using 100 bp ladder. Genetic polymorphism was estimated 
by comparing size of the bands. The $\mathrm{F}_{1} \mathrm{~S}$ that showed alleles of both the parents were termed as hybrids usually referred to as true hybrids in present study to differentiate those from putative hybrids.

\section{Results and Discussion}

Crosses between chickpea genotypes 'ICC16349 ' and 'GPF-2' generated a total of 80 putative hybrid seeds, out of which 25 were from reciprocal crosses and 55 were from direct crosses. Of the 51 primer pairs of SSRs used to amplify DNA of both the parents, only one i.e. TA 180 (1.2\% of total SSRs) showed polymorphism between the parents. Hence, TA 180 was used for confirmation of hybridity of putative hybrids (Figure 1). Out of 80 putative hybrids screened, only 34 amplified both the alleles corresponding to ICC-16349 and GPF-2 suggesting that those $34(42.5 \%)$ were true hybrids and $57.5 \%$ putative hybrids were false hybrids.

Table.1 Description of SSR markers used in the present study

\begin{tabular}{|c|c|c|c|c|}
\hline Sr No. & Primer name & Forward (5'-3') & Reverse & $\mathbf{T}_{\mathrm{m}}\left({ }^{0} \mathbf{C}\right)$ \\
\hline 1 & TA8 & AAAATTTGCACCCACAAAATATG & CTGAAAATTATGGCAGGGAAAC & 55.00 \\
\hline 2 & TA203 & ATAAAGGTTTGATCCCCATT & TGTGCATTCAGATACATGCT & 55.00 \\
\hline 3 & TR43 & AGGACGAAACTATTCAAGGTAAGTAGA & AATTGAGATGGTATTAAATGGATAACG & 55.00 \\
\hline 4 & TA30 & TCATTAAAATTCTATTGTCCTGTCCTT & ATCGTTTTTCTAAACTAAATTGTGCAT & 55.00 \\
\hline 5 & TA113 & TCTGCAAAAACTATTACGTTAATACCA & TTGTGTGTAATGGATTGAGTATCTCTT & 55.00 \\
\hline 6 & TA59 & ATCTAAAGAGAAATCAAAATTGTCGAA & GCAAATGTGAAGCATGTATAGATAAAG & 55.00 \\
\hline 7 & TA28 & TAATTGATCATACTCTCACTATCTGCC & TGGGAATGAATATATTTTTGAAGTAAA & 55.00 \\
\hline 8 & TA2 & AAATGGAAGAAGAATAAAAACGAAAC & TTCСАТТСТTТАТТАТССАТАТСАСТАСА & 55.00 \\
\hline 9 & TA146 & CTAAGTTTAATATGTTAGTCCTTAAATTAT & ACGAACGCAACATTAATTTTATATT & 55.00 \\
\hline 10 & TA72 & GAAAGATTTAAAAGATTTTCCACGTTA & TTAGAAGCATATTGTTGGGATAAGAGT & 55.00 \\
\hline 11 & TA116 & AATTCAATGACGAATTTTTATAAGGG & AAAAAGAAAAGGGAAAAGTAGGTTTTA & 55.00 \\
\hline 12 & TA130 & TCTTTCTTTGCTTCCAATGT & GTAAATCCCACGAGAAATCAA & 55.00 \\
\hline 13 & TR20 & ACCTGCTTGTTTAGCACAAT & CCGCATAGCAATTTATCTTC & 55.50 \\
\hline 14 & NCPGR209 & ATTGTTTGTTGGAGTGATGG & CACGGTTTCATTGTCTTGTT & 55.00 \\
\hline 15 & TA22 & TCTCCAACCCTTTAGATTGA & TCGTGTTTACTGAATGTGGA & 55.00 \\
\hline 16 & TA80 & CGAATTTTTACATCCGTAATG & AATCAATCCATTTTGCATTC & 55.00 \\
\hline 17 & TA176 & ATTTGGCTTAAACCCTCTTC & TTTATGCTTCCTCTTCTTCG & 55.00 \\
\hline 18 & TR44 & TTAATATTCAAAAACTCTCTTGTGCAAT & TTTACAACAGCGCTTGTATTTAGTAAG & 55.00 \\
\hline 19 & TR35 & ACTTTGGTTTAACATTTTCGGTAGTTA & AGTATCAACGTCATGTGTAACTCGTAT & 55.00 \\
\hline 20 & TR1 & CGTATGATTTTGCCGTCTAT & ACCTCAAGTTCTCCGAAAGT & 55.00 \\
\hline 21 & TA180* & CATCGTGAATATTGAAGGGT & CGGTAAATAAGTTTCCCTCC & 55.00 \\
\hline 22 & TA14 & TGACTTGCTATTTAGGGAACA & TGGCTAAAGACAATTAAAGTT & 55.00 \\
\hline 23 & TA78 & CGGTAAATAAGTTTCCCTCC & CATCGTGAATATTGAAGGGT & 55.00 \\
\hline 24 & TA64 & ATATATCGTAACTCATTAATCATCCGC & AAATTGTTGTCATCAAATGGAAAATA & 55.00 \\
\hline 25 & NCPGR264 & TGGGAATCTTGTTGGTTCTT & TGAAAGGAGATGGAAAAAGC & 57.10 \\
\hline 26 & TS43 & AAGTTTGGTCATAACACACATTCAATA & TAAATTCACAAACTCAATTTATTGGC & 55.00 \\
\hline 27 & TA5 & ATCATTTCAATTTCСТCAАСТАTGAAT & TCGTTAACACGTAATTTCAAGTAAAGAT & 55.00 \\
\hline 28 & NCPGR263 & CAAGGATGAATGTGTGTGTG & CATAGTATCCTCGGTTTCCC & 55.50 \\
\hline 29 & NCPGR136 & GGACTGAGTGAGTTCGTCTT & GTATCCTCGGTTTCCCTATC & 54.00 \\
\hline 30 & NCPGR117 & GAACTTCTTCAATCTCACGG & CTAGCACGATGAAAGGATTC & 54.50 \\
\hline 31 & NCPGR247 & CAATGATTGGTTCTCTCCTC & GGTTTGACTAAAATATGGCG & 54.50 \\
\hline 32 & NCPGR281 & GCAATGATTGGTTCTCTCCT & GTGGAATTCTTTAGGGTTTGAC & 56.50 \\
\hline 33 & NCPGR231 & АACCTCCGTCCACACATTTC & GGTCGAAGCCATTGTTTTGT & 59.40 \\
\hline 34 & NCPGR224 & TGGAATTAGTTGATGTGACAA & ATTTCCCGTGTCTTTGAGAT & 59.20 \\
\hline
\end{tabular}




\begin{tabular}{|l|l|l|r|c|}
\hline $\mathbf{3 5}$ & NCPGR214 & ATTTCCCGTGTCTTTGAGAT & GGAATTAGTTGATGTGACAATG & 54.50 \\
\hline $\mathbf{3 6}$ & NCPGR 127 & CATAATGCAAGGGCAATTAG & CTCTTATCTTCATGTTGCCG & 55.50 \\
\hline $\mathbf{3 7}$ & NCPGR 111 & AATAACTCCATTTGGCTTGA & GCGGTAATTACACAATACAGG & 54.50 \\
\hline $\mathbf{3 8}$ & NCPGR142 & TAACTCCATTTGGCTTGAGA & TAACCTTATATGGTAGGCGG & 54.50 \\
\hline $\mathbf{3 9}$ & NCPGR252 & TTGCCCTGAGGAATACATTA & GGTTGTTGAAGGCATAACTG & 54.30 \\
\hline $\mathbf{4 0}$ & NCPGR255 & TCAGTGGTATTGAGACATCG & CCATCTTCAAAAGTGAACCT & 54.00 \\
\hline $\mathbf{4 1}$ & TA25 & AGTTTAATTGGCTGGTTCTAAGATAAC & AGGATGATCTTTAATAAATCAGAATGA & 55.00 \\
\hline $\mathbf{4 2}$ & TA42 & ATATCGAAATAAATAACAACAGGATGG & TAGTTGATACTTGGATGATAACCAAAA & 55.00 \\
\hline $\mathbf{4 3}$ & GA 11 & GTTGAGCAACAAAGCCACAA & TTCTTGTCTGGTTGTGTGAGC & 55.00 \\
\hline $\mathbf{4 4}$ & TS83 & AAAAATCAGAGCCAACCAAAAA & AAGTAGGAGGCTAAATATGGAAAAGT & 55.00 \\
\hline $\mathbf{4 5}$ & TA96 & TGTTTTGGAGAAGAGTGATTC & TGTGCATGCAAATTCTTACT & 55.00 \\
\hline $\mathbf{4 6}$ & TA37 & ACTTACATGAATTATCTTTCTTGGTCC & CGTATTCAAATAATCTTTCATCAGTCA & 55.00 \\
\hline $\mathbf{4 7}$ & TA27 & GATAAAATCATTATTGGGTGTCCTTT & TTCAAATAATCTTTCATCAGTCAAATG & 55.00 \\
\hline $\mathbf{4 8}$ & NCPGR254 & GCCTTTTTCAATTCTCTCA & CCCAAAGAAGACAAAACAAC & 54.50 \\
\hline $\mathbf{4 9}$ & NCPGR261 & GATTGTGTGGCAAAATCCAT & ACTCTCAGGTTGCTGTTCTGA & 58.90 \\
\hline $\mathbf{5 0}$ & NCPGR146 & AACGTGAAATTCCACCACTA & GAGTCGATTTCGTGTTGATT & 55.40 \\
\hline $\mathbf{5 1}$ & TA96 & TGTTTTGGAGAAGAGTGATTC & TGTGCATGCAAATTCTTACT & 55.00 \\
\hline
\end{tabular}

*polymorphic primer

Fig.1 Amplification pattern of parents (ICC-16349 and GPF-2) and putative hybrids as revealed by SSR marker TA 180. Names of parents and hybrids are given at the termini of lanes. $M=100$ bp DNA ladder, $*=$ true hybrids

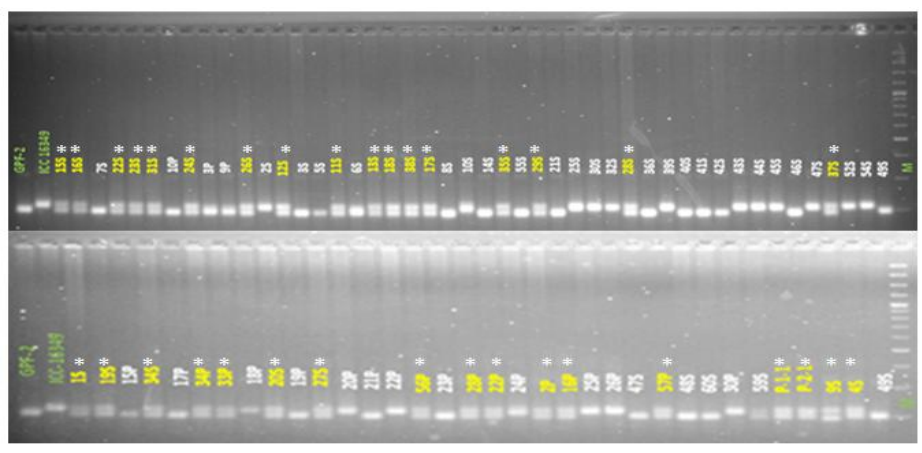

The study suggested that in chickpea breeding programmes, hybridity must be confirmed using DNA based markers to avoid inclusion of selfed plants in breeding programmes. The study further indicated that chickpea breeders usually include significant number $(>50 \%)$ of selfed plants as hybrids in breeding programmes, thereby jeopardizing the objective of chickpea improvement. Similar studies regarding hybridity confirmation were conducted by various workers. SSR markers have already been used for confirmation of hybridity in chickpea (Smitha and Katageri, 2019). They identified 13 markers which were polymorphic for both the parents i.e. Super
Annigeri-1 $\times$ BS 100B and Super Annigeri-1 $\times$ BS 72C2. However, only one marker ICCM0299 was able to detect the presence of both the parental alleles in $\mathrm{F}_{1} \mathrm{~s}$ and thus, was used for confirmation of hybridity. Morais et al., (2016) genotyped common bean with 24 microsatellite markers. Out of $342 \quad F_{1} \mathrm{~s}$ obtained from 21 different parental crosses, $325(82.91 \%)$ were confirmed as true hybrids. Johnson et al., (2019) conducted hybrid testing and studied heterosis in relation to genetic divergence in chickpea under rice based cropping system. A total of $25 \mathrm{SSR}$ markers with known sequences were used out of which only SSR21 and SSR22 were 
polymorphic between the parents. Reena and Jaiwal (2014) confirmed intra-specific and inter-specific $F_{1}$ hybrids for salt tolerance in mungbean using trait specific SSRs. Sixteen different intra-specific and inter-specific hybrid populations obtained by three type of crosses among salt susceptible and salt tolerant lines were tested for hybridity by using 15 gene specific SSRs. Only two primers i.e. SSR3435 \& SSR4041 produced polymorphism between the parents. The SSR markers in addition to hybridity confirmation have also been used for testing genetic purity in maize (Wang et al., 2002) and rice (Nandakumar et al., 2004).

Identification and characterization of hybrid cultivars is important for varietal improvement, seed production and release. For successful crop production, genetic production of hybrid seed must be maintained. Use of DNA markers offer distinct advantages over biochemical and morphological markers. Morphological markers are highly influenced by environmental factors and are time and labour consuming. Also, biochemical markers e.g. protein and isozyme markers are least affected by environment but they fail to differentiate closely related genotypes due to limited polymorphism (Luchhese et al., 1999). DNA markers overcome most drawbacks of biochemical and morphological markers and are useful for identification of hybrids.

The present study revealed that SSRs were robust and reliable markers for confirmation of hybridity in chickpea. The study also revealed that chickpea crossing might generate high proportion of selfed plants that may be designated falsely as hybrids. The study further demonstrated that putative hybrids must be confirmed by the use of SSR markers to identify true hybrids and to omit any errors of inclusion of false hybrids in breeding programme.

\section{References}

Atalay, E., and Babaoglu, M. 2012. Determination of genetic relationship in Turkish Chickpea (Cicer arietinum L.) genotypes using SSR molecular markers and capillary electrophoresis. The J. of Animal \& Plant Sci, 22 (2): 369-375.

Beckmann, J.S., and Soller, M.W. 1990. Toward a unified approach to genetic mapping of eukaryotes based on Sequence Tagged Microsatellite Sites. Nat. Biotechnol., 8: 930-932.

Gaur, R., Sethy, N.K., Choudhary, S., Shokeen, B., Gupta, V. and Bhatia, S. 2011. Advancing the STMS genomic resources for defining new locations on the intraspecific genetic linkage map of chickpea (Cicer arietinum L.). BMC Genomics, 12: 117.

Johnson, P.L., Sharma, R.N., Nanda, H.C. 2019. Hybridity testing and heterosis in relation to genetic divergence in chickpea (Cicer arietinum L.) under rice based cropping system. Indian Journal of Genetics and Plant Breeding (The)., 79 (3): 622-5.

Luchhese, C., Dinelli, G., Miggiano, A. and Lovato, A. 1999. Identification of pepper (Capsicum spp.) cultivars by field and electrophoresis tests. Seed Science \& Technology, 27: 37-47.

Maesen, V.D.L.J.G. 1987. Origin, history and taxonomy of chickpea. In: $E d s \quad M C$ Saxena and KB Singh. The Chickpea, C.A.B. International, Wallingford, UK. pp. 11-34.

Morais, S.R., Vieira, A.F., Almeida, L.C., Rodrigues, L.A., Melo, P.G., Faria, L.C., Melo, L.C., Pereira, H.S. and Souza, T.L. 2016. Application of microsatellite markers to confirm controlled crosses and assess genetic identity in common bean. Crop Breeding and Applied Biotechnology., 16 (3): 234-9. 
Murray, M.G. and Thompson, W.F. 1980. Rapid isolation of high molecular weight plant DNA Nucleic Acids Res., 8: 4321-4325.

Nandakumar, N., Singh, A.K., Sharma, R.K., Mohapatra, T., Prabhu, K.V. and Zaman, F.U. 2004. Molecular fingerprinting of hybrids and assessment of genetic purity of hybrid seeds in rice using microsatellite markers. Euphytica, 136: 257-264.

Philologos. 2005. Chickpeas-On Language. Jewish Daily Forward. http://www.forward.com/articles/2119/.

Reena, and Jaiwal, P.K. 2014. Confirmation of intra and inter-specific $\mathrm{F}_{1}$ hybrids for salt tolerance in mungbean [Vigna radiata (L). WILCZEK] genotype using trait-specific SSRs. Journal of international academic research for multidisciplinary, 2 (10): 438-450.

Smitha, S., and Katageri, S. 2019. Hybridity confirmation in chickpea (Cicer arietinum L.) through SSR molecular markers. J. Farm Sci. 33 (1): 44-48.

Tautz, D., and Renz, M. 1984. Simple sequence are ubiquitous repetitive components of eukaryotic genomes.
Nucleic Acids Res. 12 (10): 4127-4138. Varshney, R.K., Mir, R.R., Bhatia, S., Thudi, M., Hu, Y., Azam, S., Zhang, Y., Jaganathan, D., You, F.M., Gao, J., Riera-Lizarazu, O., and Ming-Cheng, L. 2014. Integrated physical, genetic and genome map of chickpea (Cicer arietinum L.). Funct. Integr. Genomics 14: 59-73.

Vavilov, N.I. 1926. Studies on the origin of cultivated plants. Bulletin of Applied Botany, Leningrad 16: 1-248.

Wang, J., Zhong, G.Y., Chin, E.C.L., Register, J.C., Riley, R.D., Nieber, W.S. and Smith, J.S.C. 2002. Identification of parents of $\mathrm{F}_{1}$ hybrids through SSR profiling of maternal and hybrid tissue. Euphytica 124: 29-34.

Winter, P., Pfaff, T., Udupa, S.M., Huttel, B., Sharma, P.C., Sahi, S., ArreguinEspinoza, R., Weigand, F., Muehlbauer, F.J. and Kahl, G. 1999. Characterization and mapping of sequence tagged microsatellite sites in the chickpea (Cicer arietinum L.) genome. Mol. Genet. 262: 90-101.

\section{How to cite this article:}

Sanchit Thakur, Jai Dev Sharma and Kamal Dev Sharma. 2020. Confirmation of Hybridity using DNA-based Markers is Essential in Chickpea (Cicer arietinum L.). Int.J.Curr.Microbiol.App.Sci. 9(11): 623-628. doi: https://doi.org/10.20546/ijcmas.2020.911.076 